\title{
Comparison of ground reaction force during gait between the nonparetic side in hemiparetic patients and the dominant side in healthy subjects
}

\author{
Dae-Hee Lee', Woo-Nam Chang ${ }^{2}$, Hye-Joo Jeon ${ }^{1, *}$ \\ 'Department of Physical Therapy, U1 University, Yeongdong, Korea \\ ${ }^{2}$ Department of Physical Therapy, Yongin University, Yongin, Korea
}

Considering the occurrence of gait impairment following stroke, walking recovery is an important goal of rehabilitation. Ground reaction force (GRF) is used for gait assessment of rehabilitation progress during exercise in stroke patients. The aim of this study was to compare the GRF during gait of the nonparetic side in hemiparetic patients and the dominant side in healthy subjects. Twenty hemiparetic patients and 20 healthy subjects were enrolled in the study. Force plate was used to evaluate GRF during gait. Additionally, with the patients and subjects in supine position, we measured their range of motion (ROM) in ankle dorsiflexion using a digital goniometer. The force values of stance phase on the nonparetic side of hemiparetic patients were significantly less than on the dominant side of healthy subjects $(P<0.05)$. The impulse values of stance phase on the paretic side and the nonparetic side of hemiparetic patients were significantly greater than on the dominant side of healthy subjects $(P<0.05)$. The ankle ROM result was significantly correlated with the GRF values $(P<0.05)$. It is important to assess and understand the nonparetic side as well as paretic side. These results suggest that the analysis of GRF for exercise rehabilitation will be a valuable clinical evaluation in hemiparetic patients after a stroke.

Keywords: Ground reaction force, Gait, Stroke, Nonparetic side, Ankle range of motion

\section{INTRODUCTION}

Gait disorders are observed to prevail in a great proportion of stroke patients and limit their ambulatory ability in the community (Keenan et al., 1984). Hemiparetic patients predominantly support their body weight with the nonparetic lower limb after the occurrence of a stroke (Bohannon and Larkin, 1985). Interlimb symmetry of weight support is an important goal of rehabilitation (Sackley, 1991). Commonly, ground reaction force (GRF) has been used for the assessment of the lower limb support function, as it has simple applicability and high accuracy (Hesse et al., 1994). GRF is an important indicator of the intensity of the force on a body during ground contact (McClay et al., 1994). To our knowledge, previous studies have investigated the relationship between walking and the GRF patterns of stroke patients (Chen et al., 2007;
Hsiao et al., 2016; Kim and Eng, 2003). The evaluation of gait in hemiparetic patients by the analysis of the paretic side is commonly used as the basis for gait treatments. However, gait analysis focuses on the rehabilitation of the paretic side suggesting that that there is a lack of evaluation of the nonparetic side.

It is suggested that severe stroke results in primary motor control problems in both the paretic side and the nonparetic side because they are compensating for the disturbed control of the contralateral side (Shiavi et al., 1987). The nonparetic limb adaptations may be the result of multiple compensatory mechanisms which would cause hemiparetic patients to adapt to their walking strategy (Chen et al., 2003; Gaviria et al., 1996). Additionally, their sedentary life contributes to the muscle atrophy on the nonparetic side (Tokuno and Eng, 2006). Indeed, a previous study has shown that considerable muscle weakness is present in the nonpa-
${ }^{*}$ Corresponding author: Hye-Joo Jeon (iD https://orcid.org/0000-0001-8491-3074 Department of Physical Therapy, U1 University, 310 Daehak-ro, Yeongdong-eup, Yeongdong-gun, Chungcheongbuk-do 29131, Korea

E-mail: bluestar@ul.ac.kr

Received: June 22, 2020 / Accepted: July 16, 2020
This is an Open Access article distributed under the terms of the Creative Commons Attribution Non-Commercial License (https://creativecommons.org/licenses/by-nc/4.0/) which permits unrestricted non-commercial use, distribution, and reproduction in any medium, provided the original work is properly cited. 
retic side of hemiparetic patients (Gerrits et al., 2009). It is indicated that the muscle weakness may cause secondary complications, as a result of corticospinal tract damage (Madhavan et al., 2011). In fact, the estimated percentage of uncrossed tracts is about $10 \%$ 20\% (Chollet et al., 1991). Thus, researchers have suggested that they could be activated with bilateral movement (Mudie and Matyas, 2000).

Despite its clinical importance, there has been little to no research on the difference between the nonparetic side in hemiparetic patients and the healthy subjects. To completely understand how persons after stroke overcome disabilities, one must understand the movement strategies used in the paretic side and the nonparetic side. It is proposed to us that the nonparetic limb function should not be neglected during exercise rehabilitation. Therefore, we conducted this study to compare the nonparetic side in hemiparetic patients with the dominant side in healthy subjects. The purpose of this study was to compare the GRF during gait of the nonparetic side in hemiparetic patients and the dominant side in healthy subjects.

\section{METERIALS AND METHODS}

\section{Participants}

A total of 40 participants (20 healthy subjects and 20 hemiparetic patients after stroke) were recruited in the study. The inclusion criteria were as follows: (a) age $<70$ years; (b) diagnosis of hemiplegia after stroke; (c) < 24 months after the occurrence of stroke; (d) ability to follow verbal commands; (e) ability to walk to $50 \mathrm{~m}$ independently without any gait aids; (f) had Modified Ashworth Scale grade lesser than 2 . The exclusion criteria were as follows: (a) lack of visual dysfunction; (b) unstable blood pressure or cardiovascular condition as determined by the physician; (c) strokes involving the brainstem or cerebellum; (d) orthopaedic problems from conditions other than stroke. Only healthy subjects with no neurological or musculoskeletal disorders affecting the lower limbs, and no history of surgery of the spine or lower limbs were considered. A total of 40 participants were randomly selected from people who responded to flyers that were placed throughout the hospital and to word of mouth. The subject characteristics are presented in Table 1. This study was approved by the Ethics Committee of Yongin University (approval number: 2-1040966-AB$\mathrm{N}-01-20)$, and all subjects provided written informed consent.

\section{0-m walk test}

Neurological patients are the most regularly studied group of
Table 1. Clinical characteristics of the participants

\begin{tabular}{lcc} 
Variable & $\begin{array}{c}\text { Hemiparetic patients } \\
(\mathrm{n}=20)\end{array}$ & $\begin{array}{c}\text { Healthy subjects } \\
(\mathrm{n}=20)\end{array}$ \\
\hline Age $(\mathrm{yr})$ & $41.50 \pm 14.59$ & $34.15 \pm 5.81$ \\
Height $(\mathrm{cm})$ & $168.23 \pm 7.97$ & $169.16 \pm 7.11$ \\
Body weight $(\mathrm{kg})$ & $69.51 \pm 12.16$ & $65.25 \pm 11.30$ \\
Sex & & \\
Male & $11(55)$ & $8(40)$ \\
Female & $9(45)$ & $12(60)$ \\
Hemiparetic and dominant side & & \\
Left & $8(40)$ & $2(10)$ \\
$\quad$ Right & $12(60)$ & $18(90)$ \\
Type of stroke & & \\
Haemorrhage & $13(65)$ & - \\
$\quad$ Infarction & $7(35)$ & - \\
Time after stroke (mo) & $7.85 \pm 7.31$ & - \\
\hline
\end{tabular}

Values are presented as mean \pm standard deviation or number (\%).

patients in studies applying walking assessments as an outcome measure (Graham et al., 2008). In this test, the individual independently walked without an assistive device for a distance of $10 \mathrm{~m}$. The time taken for the intermediate $6 \mathrm{~m}$ is measured to allow for acceleration and deceleration. It can be either tested at the preferred walking speed or the maximum walking speed. The average value of three repetitions was calculated.

\section{Vertical ground reaction force}

The subjects were requested to walk at their most natural speed along a pathway over force plates used to record the GRF. The GRF data were collected using two force plates $(464 \times 508 \times 82.5$ mm, model OR 6-7-2000, Advanced Mechanical Technologies Inc., Watertown, MA, USA) set at a sampling rate of 1,000 Hz. Three appropriate trials were collected for each limb.

\section{Range of motion in ankle joint}

The digital goniometer has appropriate concurrent criterion-related validity as a tool for the measurement of joint ROM and equivalent inter- and intrarater reliability when compared to the general goniometer (Carey et al., 2010). The ankle ROM was measured with the participant placed in a supine position on a treatment table. A low cushion was placed below the knee joint to avoid a hyperextended knee. The digital goniometer axis was aligned approximately $1.5 \mathrm{~cm}$ inferior to the lateral malleolus. The stationary arm was aligned parallel to the longitudinal axis of the fibula, lining up with the head of the fibula. The participant completed three trials per limb, with the average of each side used as 
Table 2. Comparisons of walking speed

\begin{tabular}{lcccc} 
Index $(\mathrm{m} / \mathrm{sec})$ & $\begin{array}{c}\text { Hemiparetic } \\
\text { patients }\end{array}$ & $\begin{array}{c}\text { Healthy } \\
\text { subjects }\end{array}$ & $Z$ & $P$-value \\
\hline Self-selected & $0.55 \pm 0.35$ & $1.25 \pm 0.13$ & -4.674 & $0.000^{*}$ \\
Fast-velocity & $0.81 \pm 0.50$ & $1.88 \pm 0.17$ & -5.122 & $0.000^{*}$ \\
\hline
\end{tabular}

Values are presented as mean \pm standard deviation.

${ }^{*} P<0.05$.

the representative value.

\section{Statistical analysis}

Statistical analyses were performed using IBM SPSS ver. 18.0 (IBM Co., Armonk, NY, USA). Data were expressed as means \pm standard deviations. The differences between the paretic side and the nonparetic side in hemiparetic patients were assessed by performing the Wilcoxon signed-rank test. The Mann-Whitney test was used to determine the differences between the nonparetic side in hemiparetic patients and the dominant side in healthy subjects. The Pearson correlation coefficients were used to elucidate the relationship between the GRF and ankle ROM. A $P$-value of $<0.05$ was considered as statistically significant.

\section{RESULTS}

\section{0-m walk test}

The self-selected and fast-velocity walking speeds were reduced in hemiparetic patients compared with those in healthy subjects $(P<0.05)$ (Table 2).

\section{Vertical ground reaction force}

There was no statistically significant difference in the loading response force (LRF) and loading response time (LRT) values between the paretic side and nonparetic side of hemiparetic patients $(P>0.05)$. The loading response impulse (LRI) value from the nonparetic side was significantly higher than from the paretic side of hemiparetic patients $(P<0.05)$. All mid stance force (MSF), mid stance time (MST), and mid stance impulse (MSI) values from the nonparetic side were observed to be significantly higher than from the paretic side of hemiparetic patients $(P<0.05)$. There was no statistically significant difference in the terminal stance force (TSF) and terminal stance time (TST) values between the paretic side and the nonparetic side sides of hemiparetic patients $(P>0.05)$. The terminal stance impulse (TSI) value from the nonparetic side was observed to be significantly higher than from the paretic side of hemiparetic patients $(P<0.05)$ (Table 3). The LRF value from the dominant side of healthy subjects was observed to be signifi-
Table 3. Comparisons of vertical ground reaction force between the nonparetic and the paretic sides of hemiparetic patients

\begin{tabular}{lcccc}
\hline Variable & Nonparetic side & Paretic side & $Z$ & $P$-value \\
\hline LRF $(\%$ BW) & $969.38 \pm 91.84$ & $845.16 \pm 225.69$ & -1.755 & 0.079 \\
LRT $(\%$ stride) & $27.41 \pm 5.54$ & $29.87 \pm 6.30$ & -1.382 & 0.167 \\
LRI (N-sec) & $201.47 \pm 115.45$ & $138.93 \pm 80.62$ & -2.427 & $0.015^{*}$ \\
MSF $(\%$ BW) & $864.18 \pm 126.54$ & $764.03 \pm 174.53$ & -2.689 & $0.007^{*}$ \\
MST (\% stride) & $51.39 \pm 12.68$ & $40.07 \pm 8.07$ & -2.913 & $0.004^{*}$ \\
MSI (N-sec) & $478.72 \pm 375.36$ & $205.09 \pm 98.00$ & -3.921 & $0.000^{*}$ \\
TSF $(\%$ BW) & $958.72 \pm 72.35$ & $952.52 \pm 107.80$ & -0.187 & 0.852 \\
TST (\% stride) & $69.94 \pm 3.94$ & $69.97 \pm 3.68$ & -0.299 & 0.765 \\
TSI (N-sec) & $672.52 \pm 394.05$ & $483.73 \pm 236.00$ & -3.548 & $0.000^{*}$ \\
\hline
\end{tabular}

Values are presented as mean \pm standard deviation.

LRF, loading response force; LRT, loading response time; LRI, loading response impulse; MSF, mid stance force; MST, mid stance time; MSI, mid stance impulse; TSF, terminal stance force; TST, terminal stance time; TSI, terminal stance impulse; BW, body weight.

${ }^{*} P<0.05$.

cantly higher than from the nonparetic side of hemiparetic patients $(P<0.05)$. The LRT and LRI values from the nonparetic side of hemiparetic patients were significantly higher than from the dominant side of healthy subjects $(P<0.05)$. The MTF and MSI values from the nonparetic side of hemiparetic patients were significantly higher than from the dominant side of healthy subjects $(P<0.05)$. However, there was no statistically significant difference in the MST value between the nonparetic side of hemiparetic patients and the dominant side of healthy subjects $(P>0.05)$. The TSF and the TST values from the dominant side of healthy subjects were observed to be significantly higher than from the nonparetic side of hemiparetic patients $(P<0.05)$. The TSI value from the nonparetic side of hemiparetic patients was significantly higher than from the dominant side of healthy subjects $(P<0.05)$ (Table 4$)$.

\section{Range of motion in ankle joint}

There was a statistically significant difference in ankle dorsiflexion ROM value between the paretic side and nonparetic side of hemiparetic patients $(P<0.05)$ (Table 5). Additionally, there was a statistically significant difference in the dorsiflexion $\mathrm{ROM}$ value between the dominant side of healthy subjects and the nonparetic side of hemiparetic patients $(P<0.05)$ (Table 6 ).

\section{Correlations between the ground reaction force and ankle range of motion}

There was a significant correlation between the LRF value and the ankle dorsiflexion $\mathrm{ROM}(r=0.512, P<0.05)$. There was a significant correlation between the LRT value and the ankle dorsi- 
Table 4. Comparisons of vertical ground reaction force between the nonparetic side of hemiparetic patients and the dominant side of healthy subjects

\begin{tabular}{lcccc}
\hline Variable & Nonparetic side & Dominant side & $Z$ & $P$-value \\
\hline LRF $(\%$ BW $)$ & $969.38 \pm 91.84$ & $1,098.46 \pm 83.62$ & -1.999 & $0.000^{*}$ \\
LRT $(\%$ stride) & $27.41 \pm 5.54$ & $23.89 \pm 3.88$ & -1.512 & $0.033^{*}$ \\
LRI (N-sec) & $201.47 \pm 115.45$ & $79.33 \pm 26.86$ & -2.625 & $0.000^{*}$ \\
MSF $(\%$ BW) & $864.18 \pm 126.54$ & $843.38 \pm 74.29$ & -2.833 & $0.035^{*}$ \\
MST (\% stride) & $51.39 \pm 12.68$ & $46.15 \pm 3.68$ & -3.077 & 0.433 \\
MSI (N-sec) & $478.72 \pm 375.36$ & $173.63 \pm 46.53$ & -4.015 & $0.000^{*}$ \\
TSF $(\%$ BW) & $958.72 \pm 72.35$ & $1,106.32 \pm 62.38$ & -0.122 & $0.000^{*}$ \\
TST (\% stride) & $69.94 \pm 3.94$ & $74.28 \pm 3.06$ & -0.017 & $0.002^{*}$ \\
TSI (N-sec) & $672.52 \pm 394.05$ & $295.16 \pm 73.26$ & -3.668 & $0.000^{*}$
\end{tabular}

Values are presented as mean \pm standard deviation.

LRF, loading response force; LRT, loading response time; LRI, loading response impulse; MSF, mid stance force; MST, mid stance time; MSI, mid stance impulse; TSF terminal stance force; TST, terminal stance time; TSI, terminal stance impulse; BW, body weight

${ }^{*} P<0.05$.

Table 5. Comparison of range of motion in ankle dorsiflexion between the nonparetic side and the paretic side of hemiparetic patients

\begin{tabular}{lcccc}
\hline Index (degree) & Nonparetic side & Paretic side & $Z$ & $P$-value \\
\hline Dorsiflexion range of motion & $11.1 \pm 3.93$ & $3.04 \pm 3.45$ & -3.921 & $0.000^{*}$ \\
\hline
\end{tabular}

Values are presented as mean \pm standard deviation.

${ }^{*} P<0.05$.

flexion ROM $(r=-0.342, P<0.05)$. There was a significant correlation between the LRI value and the ankle dorsiflexion ROM $(r=0.391, P<0.05)$.

There was no significant correlation between MSF value and ankle dorsiflexion ROM $(r=0.222, P>0.05)$. There was a significant correlation between the MST value and the ankle dorsiflexion ROM $(r=0.302, P<0.05)$. There was no significant correlation between the MSI value and the ankle dorsiflexion ROM $(r=$ $-0.156, P>0.05)$. There was a strong and significant correlation between the TSF value and the ankle dorsiflexion ROM $(r=0.530$, $P<0.05)$. There was a significant correlation between the TST value and the ankle dorsiflexion $\operatorname{ROM}(r=0.539, P<0.05)$. There was a significant correlation between the TSI value and the ankle dorsiflexion $\operatorname{ROM}(r=-0.405, P<0.05)$ (Table 7).

\section{DISCUSSION}

In this study, the vertical GRF values were evaluated and compared to analyse the differences between the hemiparetic patients and healthy subjects. A common GRF was a bimodal M form because the vertical force applied during the initial contact and push-
Table 6. Comparison of range of motion in ankle dorsiflexion between the nonparetic side of hemiparetic patients and the dominant side of healthy subjects

\begin{tabular}{lcccc}
\hline Index (degree) & Nonparetic side & Dominant side & $Z$ & $P$-value \\
\hline Dorsiflexion range of motion & $11.1 \pm 3.93$ & $19.09 \pm 1.06$ & -5.426 & $0.000^{*}$
\end{tabular}

Values are presented as mean \pm standard deviation

${ }^{*} P<0.05$.

Table 7. Correlation between the vertical ground reaction force and range of motion in ankle dorsiflexion

\begin{tabular}{lcc}
\hline Variable & Dorsiflexion range of motion (R-value) & $P$-value \\
\hline LRF $(\% B W)$ & 0.512 & $0.000^{*}$ \\
LRT $(\%$ stride) & -0.342 & $0.001^{*}$ \\
LRI (N-sec) & -0.391 & $0.000^{*}$ \\
MSF (\%BW) & 0.222 & 0.754 \\
MST (\% stride) & 0.302 & $0.009^{*}$ \\
MSI (N-sec) & -0.156 & $0.009^{*}$ \\
TSF $\%$ BW) & 0.530 & $0.000^{*}$ \\
TST (\% stride) & 0.539 & $0.000^{*}$ \\
TSI (N-sec) & -0.405 & $0.000^{*}$ \\
\hline
\end{tabular}

LRF, loading response force; LRT, loading response time; LRI, loading response impulse; MSF, mid stance force; MST, mid stance time; MSI, mid stance impulse; TSF, terminal stance force; TST, terminal stance time; TSI, terminal stance impulse; BW, body weight.

${ }^{*} P<0.05$

off in the stance phase exceeds body weight, while those applied during the midstance were lesser than the body weight (Murray et al., 1969). Several hemiparetic patients may lose the heel-strike and push-off mechanism, changing the GRF form from the bimodal $\mathrm{M}$ form to an irregular form (Chen et al., 2007).

Our results show that the LRF and TSF values were different between hemiparetic patients and healthy subjects as the shock absorption on the heels in the initial contact and push-off in the phase does not work properly in hemiparetic patients. Individuals with poststroke hemiparesis often show reduced muscle activation of the paretic limb during walking (Burridge et al., 2001). There is sufficient evidence that the muscle weakness is reflected by the inability of stroke patients to generate normal muscle force (Bourbonnais and Vanden Noven, 1989).

In the present study, the RLT value indicated the later time both the paretic side and the nonparetic side in hemiparetic patients compared with healthy subjects, whereas the TST value indicated the earlier time. Based on these results, the hemiparetic patients cannot apply sufficient weight shift on both the paretic side and nonparetic side. Thus, they require additional time in the stance phase than the healthy subjects. The impulse values of both 
the paretic side and nonparetic side of hemiparetic patients were greater than those of the dominant side of healthy subjects; they were significantly greater on the nonparetic side than on the paretic side. These findings were consistent with earlier results, in which the nonparetic side showed greater impulse values than the paretic side (Horvath et al., 2001). This may explain why a proper weight was not loaded on the paretic side earlier. In addition, the reason is that additional time was needed to forward the weakened paretic limb during the swing phase. This creates difficulties such as the loading of overweight on the nonparetic side. There are differences between the performance of a nonparetic limb and a healthy limb (Olney et al., 1991; Olney et al., 1994; Parvataneni et al., 2007). For example, muscle activity is increased on the nonparetic limb during gait. It may not have a positive muscle activity on walking and might propose impairments (Raja et al., 2012). In addition, stroke patients often show changes in spatiotemporal properties during walking (Patterson et al., 2008). Such spatiotemporal asymmetries may be due to the compensatory strategies used by either the paretic limb, or the nonparetic limb (Chen et al., 2005). This can negatively affect walking ability (Olney and Richards, 1996). Thus, to improve walking ability, it is essential to not only understand the movement of the paretic limb, but to also understand the movement of the nonparetic limb. This study is clinically significant since we compared the nonparetic side in hemiparetic patients with the dominant side in healthy subjects.

Many previous studies have analyzed the relationship between the ankle and gait in stroke patients (Kitatani et al., 2016; Lamontagne et al., 2000; Lamontagne et al., 2002). Ankle dorsiflexion ROM is a crucial factor for the gait cycle (Dobkin et al., 2004). Therefore, we measured ROM in ankle dorsiflexion. The results showed that the paretic ankle dorsiflexion ROM was smaller than the nonparetic ankle dorsiflexion ROM in hemiparetic patients. It was consistent with the results of previous studies (Chung et al., 2004; Lamontagne et al., 2000; Lin et al., 2006). Decreased ankle dorsiflexion ROM of the paretic side can be caused by many factors, such as passive stiffness and spasticity of the plantar flexors (Dietz et al., 1981). Notably, the ankle dorsiflexion ROM of the nonparetic side was significantly smaller than that of the dominant side of healthy subjects in this study. It has been proposed that the ipsilaterally mediated effects from the neurological lesion may contribute to the ample muscle coactivation. The coactivation of the nonparetic side mainly during the stance phase had been indicated (Shiavi et al., 1987). It is likely that the increased muscle coactivation levels reported on the nonparetic side support postural stability during gait (Lamontagne et al., 2002). Though benefi- cial for postural stability, the increased coactivation reduces the effectiveness of movement and may produce part of the higher energy cost of locomotion reported after stroke (Zamparo et al., 1995). Limited dorsiflexion can change the foot positioning in weight bearing and decreased capacity to shift the center of gravity during standing or gait (Lin et al., 2006). In particular, the major compensations for limited dorsiflexion would include the hyperextension of the knee joint and flexion of the hip joint. Subsequently, the GRF vector would be in a backward direction of the hip joint. Therefore, these patients have an impaired dynamic balance during standing or gait (An and Jo, 2017). In the standard gait, the plantarflexor passive component controls the ankle dorsiflexion and assists the forward propulsion of the body at push-off (Hof et al., 1983).

Even though our results are not sufficient to generalize the correlation because of our small sample size, they demonstrate a strong correlation between the GRF during gait and ankle ROM. Ankle dorsiflexion ROM and plantarflexor peak torque are related and appear to be significant factors that contribute to ankle plantarflexor power and moments during walking. This suggests that increasing platarflexor peak torque and ankle dorsiflexion ROM may help increase gait ability and speed (Mueller et al., 1995). In addition, earlier studies showed some significant correlation between ankle plantarflexor and GRF (Chen et al., 2007; Lamontagne et al., 2000; Turns et al., 2007). We surmise that lesser ankle dorsiflexion ROM will make it difficult for appropriate power on the plantarflexor during the push-off. Maintaining proper conditions for ankle mobility may promote the dorsiflexion during gait, which aids the ankle push-off at the end of the stance phase of the gait.

We believe that despite the fact that the sample size was too small for generalization, the results provide a significant starting point for the future measurement of the relationship between the GRF and ankle ROM. Therefore, the results of this study must be confirmed with a larger sample size. This study also had other limitations to consider when interpreting the results. The position and the size of the force plate were not suitable for individuals with a step length.

In conclusion, there was a mostly significant difference in the nonparetic side of hemiparetic patients and the dominant side of healthy subjects. The results from our study can be used to aid exercise rehabilitation programs that solve the problems of the nonparetic side as well as the paretic side. These assessments can be identified based on a patient's status, thus helping therapists to choose the best exercise programs for the rehabilitation of stroke patients. 


\section{CONFLICT OF INTEREST}

No potential conflict of interest relevant to this study was reported.

\section{REFERENCES}

An CM, Jo SO. Effects of talocrural mobilization with movement on ankle strength, mobility, and weight-bearing ability in hemiplegic patients with chronic stroke: a randomized controlled trial. J Stroke Cerebrovasc Dis 2017;26:169-176.

Bohannon RW, Larkin PA. Lower extremity weight bearing under various standing conditions in independently ambulatory patients with hemiparesis. Phys Ther 1985;65:1323-1325.

Bourbonnais D, Vanden Noven S. Weakness in patients with hemiparesis. Am J Occup Ther 1989;43:313-319.

Burridge JH, Wood DE, Taylor PN, McLellan DL. Indices to describe different muscle activation patterns, identified during treadmill walking, in people with spastic drop-foot. Med Eng Phys 2001;23:427-434.

Carey MA, Laird DE, Murray KA, Stevenson JR. Reliability, validity, and clinical usability of a digital goniometer. Work 2010;36:55-66.

Chen CL, Chen HC, Tang SF, Wu CY, Cheng PT, Hong WH. Gait performance with compensatory adaptations in stroke patients with different degrees of motor recovery. Am J Phys Med Rehabil 2003;82:925935.

Chen CY, Hong PW, Chen CL, Chou SW, Wu CY, Cheng PT, Tang FT, Chen HC. Ground reaction force patterns in stroke patients with various degrees of motor recovery determined by plantar dynamic analysis. Chang Gung Med J 2007;30:62-72.

Chen G, Patten C, Kothari DH, Zajac FE. Gait deviations associated with post-stroke hemiparesis: improvement during treadmill walking using weight support, speed, support stiffness, and handrail hold. Gait Posture 2005;22:57-62.

Chollet F, DiPiero V, Wise RJ, Brooks DJ, Dolan RJ, Frackowiak RS. The functional anatomy of motor recovery after stroke in humans: a study with positron emission tomography. Ann Neurol 1991;29:63-71.

Chung SG, Van Rey E, Bai Z, Roth EJ, Zhang LQ. Biomechanic changes in passive properties of hemiplegic ankles with spastic hypertonia. Arch Phys Med Rehabil 2004;85:1638-1646.

Dietz V, Quintern J, Berger W. Electrophysiological studies of gait in spasticity and rigidity. Evidence that altered mechanical properties of muscle contribute to hypertonia. Brain 1981;104:431-449.

Dobkin BH, Firestine A, West M, Saremi K, Woods R. Ankle dorsiflexion as an fMRI paradigm to assay motor control for walking during rehabilitation. Neuroimage 2004;23:370-381.
Gaviria M, D'Angeli M, Chavet P, Pelissier J, Peruchon E, Rabischong P. Plantar dynamics of hemiplegic gait: a methodological approach. Gait Posture 1996;4:297-305.

Gerrits KH, Beltman MJ, Koppe PA, Konijnenbelt H, Elich PD, de Haan A, Janssen TW. Isometric muscle function of knee extensors and the relation with functional performance in patients with stroke. Arch Phys Med Rehabil 2009;90:480-487.

Graham JE, Ostir GV, Fisher SR, Ottenbacher KJ. Assessing walking speed in clinical research: a systematic review. J Eval Clin Pract 2008;14:552562.

Hesse SA, Jahnke MT, Bertelt CM, Schreiner C, Lücke D, Mauritz KH. Gait outcome in ambulatory hemiparetic patients after a 4-week comprehensive rehabilitation program and prognostic factors. Stroke 1994; 25:1999-2004.

Hof AL, Geelen BA, Van den Berg J. Calf muscle moment, work and efficiency in level walking; role of series elasticity. J Biomech 1983;16:523537.

Horvath M, Tihanyi T, Tihanyi J. Kinematic and kinetic analyses of gait patterns in hemiplegic patients. Facta Univ Series: Phys Educ Sport 2001;1:25-35

Hsiao H, Awad LN, Palmer JA, Higginson JS, Binder-Macleod SA. Contribution of paretic and nonparetic limb peak propulsive forces to changes in walking speed in individuals poststroke. Neurorehabil Neural Repair 2016;30:743-752.

Keenan MA, Perry J, Jordan C. Factors affecting balance and ambulation following stroke. Clin Orthop Relat Res 1984;(182):165-171.

Kim CM, Eng JJ. Symmetry in vertical ground reaction force is accompanied by symmetry in temporal but not distance variables of gait in persons with stroke. Gait Posture 2003;18:23-28.

Kitatani R, Ohata K, Sato S, Watanabe A, Hashiguchi Y, Yamakami N, Sakuma K, Yamada S. Ankle muscle coactivation and its relationship with ankle joint kinematics and kinetics during gait in hemiplegic patients after stroke. Somatosens Mot Res 2016;33:79-85.

Lamontagne A, Malouin F, Richards CL. Contribution of passive stiffness to ankle plantarflexor moment during gait after stroke. Arch Phys Med Rehabil 2000;81:351-358.

Lamontagne A, Malouin F, Richards CL, Dumas F. Mechanisms of disturbed motor control in ankle weakness during gait after stroke. Gait Posture 2002;15:244-255.

Lin PY, Yang YR, Cheng SJ, Wang RY. The relation between ankle impairments and gait velocity and symmetry in people with stroke. Arch Phys Med Rehabil 2006;87:562-568.

Madhavan S, Krishnan C, Jayaraman A, Rymer WZ, Stinear JW. Corticospinal tract integrity correlates with knee extensor weakness in chronic stroke survivors. Clin Neurophysiol 2011;122:1588-1594. 
McClay IS, Robinson JR, Andriacchi TP, Frederick EC, Gross T, Martin P, Valiant G, Williams KR, Cavanagh PR. A profile of ground reaction forces in professional basketball. J Appl Biomech 1994;10:222-236.

Mudie MH, Matyas TA. Can simultaneous bilateral movement involve the undamaged hemisphere in reconstruction of neural networks damaged by stroke? Disabil Rehabil 2000;22:23-37.

Mueller MJ, Minor SD, Schaaf JA, Strube MJ, Sahrmann SA. Relationship of plantar-flexor peak torque and dorsiflexion range of motion to kinetic variables during walking. Phys Ther 1995;75:684-693.

Murray MP, Seireg AH, Scholz RC. A survey of the time, magnitude and orientation of forces applied to walking sticks by disabled men. Am J Phys Med 1969;48:1-13.

Olney SJ, Griffin MP, McBride ID. Temporal, kinematic, and kinetic variables related to gait speed in subjects with hemiplegia: a regression approach. Phys Ther 1994;74:872-885.

Olney SJ, Griffin MP, Monga TN, McBride ID. Work and power in gait of stroke patients. Arch Phys Med Rehabil 1991;72:309-314.

Olney SJ, Richards C. Hemiparetic gait following stroke. Part I: characteristics. Gait Posture 1996;4:136-148.

Parvataneni K, Olney SJ, Brouwer B. Changes in muscle group work associated with changes in gait speed of persons with stroke. Clin Bio- mech (Bristol, Avon) 2007;22:813-820.

Patterson KK, Parafianowicz I, Danells CJ, Closson V, Verrier MC, Staines WR, Black SE, Mcllroy WE. Gait asymmetry in community-ambulating stroke survivors. Arch Phys Med Rehabil 2008;89:304-310.

Raja B, Neptune RR, Kautz SA. Coordination of the nonparetic leg during hemiparetic gait: expected and novel compensatory patterns. Clin Biomech (Bristol, Avon) 2012;27:1023-1030.

Sackley CM. Falls, sway, and symmetry of weight-bearing after stroke. Int Disabil Stud 1991;13:1-4.

Shiavi R, Bugle HJ, Limbird T. Electromyographic gait assessment, Part 2: preliminary assessment of hemiparetic synergy patterns. J Rehabil Res Dev 1987;24:24-30.

Tokuno CD, Eng JJ. Gait initiation is dependent on the function of the paretic trailing limb in individuals with stroke. Gait Posture 2006;24:424428.

Turns LJ, Neptune RR, Kautz SA. Relationships between muscle activity and anteroposterior ground reaction forces in hemiparetic walking. Arch Phys Med Rehabil 2007;88:1127-1135.

Zamparo P, Francescato MP, De Luca G, Lovati L, di Prampero PE. The energy cost of level walking in patients with hemiplegia. Scand J Med Sci Sports 1995;5:348-352. 